\title{
Non-differentiability and fractional differentiability on timescales
}

Received: 24 April 2017 / Accepted: 11 September 2017 / Published online: 23 September 2017

(C) The Author(s) 2017. This article is an open access publication

\begin{abstract}
This work deals with concepts of non-differentiability and a non-integer order differential on timescales. Through an investigation of a local non-integer order derivative on timescales, a mean value theorem (a fractional analog of the mean value theorem on timescales) is presented. Then, by illustrating a vanishing property of this derivative, its objectivity is discussed. As a first-hand result, the potentials and capability of this fractional derivative connected to nonsmooth analysis, including non-differentiable paths and a class of self-similar fractals, are stated. It is stated that the non-integer order derivative never vanishes almost everywhere. It has been shown that with the help of changing the order of differentiability on a q-timescale, the non-differentiability disappears.
\end{abstract}

Mathematics Subject Classification $26 \mathrm{E} 70 \cdot 26 \mathrm{~A} 33 \cdot 28 \mathrm{~A} 80$

M. Nategh $(\bowtie)$

Department of Mathematics and Statistics, Missouri S \& T, Rolla, MO 65401, USA

E-mail: nateghm@mst.edu

M. Nategh $\cdot$ A. Neamaty

Department of Mathematics and Statistics, University of Mazandaran, Babolsar, Iran

E-mail: m.nateghp@yahoo.com

A. Neamaty

E-mail: namaty@umz.ac.ir

B. Agheli

Department of Mathematics, Qaemshahr Branch, Islamic Azad University, Qaemshahr, Iran

E-mail: b.agheli@qaemiau.ac.ir 


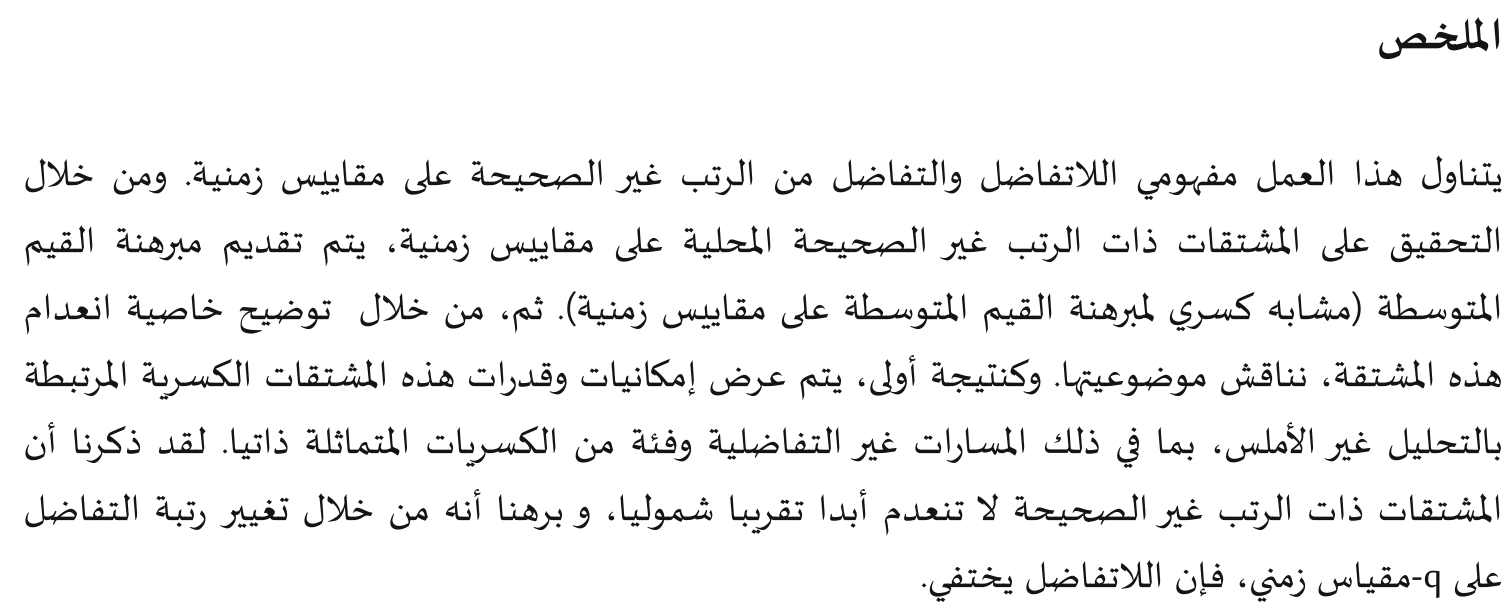

\section{Introduction}

Calculus on timescales originally was proposed in S. Hilger's Ph.D. thesis, which aimed to unify the differential and difference calculus $[15,16]$. Timescales, as closed subsets of the real line, include diverse patterns such as non-countable self-similar sets, primes and closed intervals as well. Although its fabric is one dimensional, up to an appropriate transformation, it is potentially capable of including higher dimensional patterns (see Sect. 2.1). Despite the complexity of the diverse scattering patterns in timescales, dynamic equations and inequalities on timescales are enriched by many fruitful results, and indeed research topics in this area are of great interests $[4,8,9]$.

Compared to the classical calculus with some of its nonlocal fractional counterparts with the same antiquity, a few incomplete efforts aiming to present local fractional calculus [3] or non-local fractional calculus [2,26] on timescales have been done.

Differentiation and/or integration on fractals are reported in [7,12,20,27]. In conjunction with the main result of the present work, it is noteworthy that [27] introduces a local non-integer order calculus on Cantor set, which (in its general topological definition) is a prototype of a famous and mostly observed pattern. The mentioned theory in [27] is thrived to obtain an almost comprehensive achievement in non-integer order dynamic equations on Cantor set in the local sense. Surprisingly, this local non-integer order theory studies a subclass of nondifferentiable functions including Lebesgue-Cantor function.

Based on the idea in [7], a non-integer order derivative is in accordance with the Hausdorff dimension of a compact fractal. A rigorous mathematical integration theory on fractals can be found in [12], in which the concept of a generalized Riemann integral based on the staircase function is introduced. Moreover, for a given compact fractal $F$ and a function $f: F \rightarrow \mathbb{R}$, it proposes a derivative at $t \in F$ by (provided the limit exists)

$$
\lim _{\substack{s \rightarrow t \\ s \in F}} \frac{f(s)-f(t)}{* S_{F}^{\alpha}(s)-{ }^{*} S_{F}^{\alpha}(t)},
$$

where ${ }^{*} S_{F}^{\alpha}$ is the staircase function corresponding to $F$, and $0<\alpha<1$ is the ${ }^{*} \gamma$-dimension of $F$ [12].

A timescale approach toward the differentiation theory on fractals is suggested in [3]. Indeed, [3] proposes a non-integer order differentiation on timescales that contains compact fractals as well. In this approach, an implicit assumption is that all timescales are subject to the local non-integer order differentiation theory. In other words, for a given function defined on an arbitrary timescale, the differential of that function is not equivalent to zero necessarily.

As it will be discussed in this paper, in the above-mentioned proposal, all those timescales whose set of dense points are non-empty must be excluded from the theory since the derivative of a smooth function vanishes at the dense points. To overcome the lack of applicability in the presence of this vanishing property, one may take non-differentiable functions into account and this supposition leads us to the main result of the present work.

This work is organized as follows:

Section 2 is devoted to preliminaries on timescales with $\Delta$ and $\alpha$-derivative. In Sect. 2.1, a class of selfsimilar fractals as compact timescales are studied. Non-integer order mean value on timescales is stated in 
Sect. 2.2. The mentioned theorem is a breakthrough in both integer and non-integer order differentiation and integration theory on timescales, whereas it is utilized to obtain the main background for integration theory [5]. In Sect. 3, we obtain the vanishing property of $\alpha$-derivative for smooth functions in the sense that $f^{(\alpha)}$ vanishes at right-dense points. Then, utilizing the non-integer order mean value theorem, we obtain the main result for nondifferentiable and $\alpha$-differentiable functions. Section 4 is devoted to some examples of nondifferentiable functions.

In accordance with a classical nowhere differentiability and the classical Weierstrass function, a class of $\Delta$-nondifferentiable functions on a q-timescale is introduced. Then, the $\alpha$-differentiability is represented. The specific series form of those functions is inspired by the trigonometric series form of their classical archetype, namely the Weierstrass function. Also, the q-version of a non-differentiable function in the above sense appears as a q-integral, which is a summation consisting of a finite number of terms.

\section{Preliminary concepts}

For comprehensive discussions on the calculus and dynamic equations on timescales, see [5].

Timescales are closed subsets of the real line. Some of the prevalent examples are $\mathbb{Z}, h \mathbb{Z}(h>0)$, Primes, $2^{\mathbb{Z}}$ and $\mathbb{R}$.

Three basic functions denoted by $\sigma, \rho$ and $\mu$, respectively, are defined by

$$
\begin{gathered}
\sigma(t):=\inf \{s \mid s>t\}, \quad \rho(t):=\sup \{s \mid s<t\}, \\
\mu(t):=\sigma(t)-t .
\end{gathered}
$$

A right-scattered point $t \in \mathbb{T}$ is a point for which we have $\mu(t)>0$, and it is called right-dense when $\mu(t)=0$. $\mathbb{T}^{\kappa}$ is defined by $\mathbb{T} \backslash\left\{t_{0}\right\}$, where $t_{0}$ is the left-scattered maximum. A function $f: \mathbb{T} \rightarrow \mathbb{R}$ is called regulated when its left-sided limits exist at left-dense points and its right-sided limits exist at right-dense points. It is clear that regulated functions are bounded on bounded subsets of $\mathbb{T}$. A function $f: \mathbb{T}^{\kappa} \rightarrow \mathbb{R}$ is said to be rd-continuous, if it is continuous at right-dense points, and the left-sided limits on left-dense points exist. The notation $C_{r d}(\mathbb{T})$ or simply $C_{r d}$ is served as the set of rd-continuous functions.

\subsection{Self-similar compact timescales}

The notion of timescales and non-integer order dynamic may be supported by fractal geometry. In view of the prevalence of fractal patterns in nature, some inspiring studies, specially in biology, are reported $[1,13,14]$. The present subsection is inspired by Fig. 1, [14].

Suppose $S \subset \mathbb{R}^{N}$ and $X=\left\{\left(a_{n}\right)_{n} \mid a_{n} \in \mathbb{Z}_{p}\right\}$ ( $p \in$ Primes), $X_{1} \subset X$ be non-voided and assume that there is a one to one correspondence between $S$ and $X_{1}$. Making use of $p$-based expansion of numbers, $[-1,1] / \sim$ consists of equivalence classes $t \in \frac{\mathbb{R}}{\mathbb{Z}}$, with $t_{1} \sim t_{2}$ iff $a_{n}-b_{n}=0 \bmod p$, where $t_{1}=\sum_{n} \frac{a_{n}}{p^{n}}$ and $t_{2}=\sum_{n} \frac{b_{n}}{p^{n}}$. Define $T: X \rightarrow \frac{\mathbb{R}}{\mathbb{Z}}$ by $T\left(a_{n}\right)_{n}=\sum_{n} \frac{a_{n}}{p^{n}}$, then $(X, d)$ is an infinite dimensional vector space over $\mathbb{Z}_{p}$, where $d\left(\left(a_{n}\right)_{n},\left(b_{n}\right)_{n}\right)=T\left(a_{n}-b_{n}\right)_{n}$. It is clear that $\mathbb{T}=T X_{1}$ is a timescale provided $\left(X_{1}, d\right)$ is closed in $(X, d)$.

For the next statement, members of $s \in S$ are denoted by the same notations as their counterparts in $X$, i.e., $s=\left\{a_{n}\right\}$, so $d\left(s_{1}, s_{2}\right)$ for $s_{1}, s_{2} \in S$ is defined by $d\left(\left\{a_{n}\right\},\left\{b_{n}\right\}\right)$, where $\left\{a_{n}\right\}$ and $\left\{b_{n}\right\}$ are correspondent $X$ members for $s_{1}$ and $s_{2}$.

Proposition 2.1 Let $g: S \rightarrow S$ be a function and suppose

$$
d\left(g\left(s_{1}\right), g\left(s_{2}\right)\right)=\lambda d^{\alpha}\left(s_{1}, s_{2}\right)
$$

for some $\alpha \in[0,1]$ and $0<\lambda<1$. If $S$ is correspondent to $X_{1} \subset X$, then a similar identity holds for $T \circ g \circ T^{-1}: \mathbb{T} \rightarrow \mathbb{T}$, where $\mathbb{T}=T\left(X_{1}\right) \subset \frac{\mathbb{R}}{\mathbb{Z}}$

Proof Let $t_{1}=\sum_{n \in \mathbb{N}_{0}} \frac{a_{n}}{p^{n}}$ and $t_{2}=\sum_{n \in \mathbb{N}_{0}} \frac{b_{n}}{p^{n}}$, then, $T^{-1}\left(t_{1}\right)$ and $T^{-1}\left(t_{2}\right)$ are $X$-correspondent pre-images of $t_{1}, t_{2}$ in $S$. Let $\left\{\tilde{a}_{n}\right\}$ and $\left\{\tilde{b}_{n}\right\}$ be correspondent to $g\left(s_{1}\right)$ and $g\left(s_{2}\right)$, then

$$
\left|T \circ g \circ T^{-1}\left(t_{1}\right)-T \circ g \circ T^{-1}\left(t_{1}\right)\right|=\left|T\left(g\left(s_{1}\right)-g\left(s_{2}\right)\right)\right|
$$




$$
\begin{aligned}
& =\left|\sum_{n \in \mathbb{N}_{0}} \frac{\tilde{a}_{n}-\tilde{b}_{n}}{p^{n}}\right| \\
& =d\left(\left\{\tilde{a}_{n}\right\},\left\{\tilde{b}_{n}\right\}\right) \\
& =\lambda d^{\alpha}\left(\left\{a_{n}\right\},\left\{b_{n}\right\}\right) \\
& =\lambda\left(\sum_{n \in \mathbb{N}_{0}} \frac{a_{n}-b_{n}}{p^{n}}\right)^{\alpha} \\
& =\lambda\left|t_{1}-t_{2}\right|^{\alpha} .
\end{aligned}
$$

Remark 2.2 The result of Proposition 2.1 holds if we substitute the equality sign by $\leq$.

Let $\alpha=1$ and Eq. (2.3) with $\leq$ holds for $\left\{g_{1}, g_{2}, \ldots, g_{n}\right\}$, with $0<\lambda_{i}<1$, then there exists a compact subset $S^{*} \subset S$ such that $S^{*}=\bigcup_{i=1}^{n} g_{i}\left(S^{*}\right)$ (see Chapter 9, [11]). According to Proposition 2.1, the same holds for $\left\{T \circ g_{1} \circ T^{-1}, T \circ g_{2} \circ T^{-1}, \ldots, T \circ g_{n} \circ T^{-1}\right\}$, i.e., there exists a compact subset $\mathbb{T}^{*} \subset \mathbb{T}$, for which $\mathbb{T}^{*}=\bigcup_{i=1}^{n} T \circ g_{i} \circ T^{-1}\left(\mathbb{T}^{*}\right)$. Suppose the open set condition holds in the sense that there exists an open $U \subset \mathbb{R}^{2}$ such that $\bigcup_{i=1}^{n} g_{i}(U) \subset U$ with disjoint $g_{i}(U)$ s (accordingly, $\bigcup_{i=1}^{n} T \circ g_{i} \circ T^{-1}(V) \subset V$, for some open $V \subset \frac{\mathbb{R}}{\mathbb{Z}}$ with disjoint $T \circ g_{i} \circ T^{-1}(V)$ s). If $\gamma=\operatorname{dim}_{\mathcal{H}}\left(S^{*}\right)$ denotes the Hausdorff dimension of $S^{*}$ (see Chapter 2, [11]), then by Theorem 9.3 [11], $\sum_{i=1}^{n} \lambda_{i}^{\gamma}=1$.

Using the scaling property (Sec 2.1, [11]) for $0<\alpha<1$, we obtain a similar result, while for the corresponding compact invariant set $S^{*}$ when $\alpha \neq 1$, the exponent $\gamma$ should be substituted by $\frac{\gamma}{\alpha}$ and we have $\sum_{i=1}^{n} \lambda_{i}^{\frac{\gamma}{\alpha}}=1$.

\section{$2.2 \Delta$-Calculus and fractional calculus on timescales}

Calculus on timescales begins with the following definition:

Definition 2.3 [5] Suppose $X$ is a real Banach space, $f: \mathbb{T} \rightarrow X$ is a function and $t \in \mathbb{T}^{\kappa}$. We define $f^{\Delta}(t) \in X$ to be a vector-if it exists-with the property that, for any $\epsilon>0$, there is a neighborhood $U:=(t-\delta, t+\delta)$ for some $\delta>0$ such that

$$
\left|f(\sigma(t))-f(s)-(\sigma(t)-s) f^{\Delta}(t)\right| \leq \epsilon|\sigma(t)-s| .
$$

In case $\mu(t)>0$ and $f$ is continuous, then $f$ is differentiable at $t$ and we have

$$
f^{\Delta}(t)=\frac{f(\sigma(t))-f(t)}{\mu(t)},
$$

and when the point $t$ is right-dense, $f$ is differentiable at $t$ iff the limit

$$
f^{\Delta}(t):=\lim _{s \longrightarrow t} \frac{f(t)-f(s)}{t-s}
$$

exists.

For $D \subset \mathbb{T}^{\kappa}$, a continuous function $f: \mathbb{T} \rightarrow X$ is called pre-differentiable with region of differentiation $D$, if $\mathbb{T} \backslash D$ is countable and contains no right-scattered point and also $f^{\Delta}(t)$ exists for all $t \in D$.

A non-integer order derivative in the local sense has been suggested in [21].

Definition 2.4 Let $f$ be a real-valued function defined on $\mathbb{T}, t \in \mathbb{T}^{\kappa}$ and $\alpha \in(0,1] \cap\left\{\frac{n}{2 m+1} \mid n, m \in \mathbb{N}\right\}$, the real value $f^{(\alpha)}(t)$ (if it exists) is defined to be a number with the property that, for any positive $\epsilon$, there exists a neighborhood $\mathcal{U} \subset \mathbb{T}$ of $t$ of length $\delta$ so that

$$
\left|f(\sigma(t))-f(s)-f^{(\alpha)}(t)(\sigma(t)-s)^{\alpha}\right| \leq \epsilon|\sigma(t)-s|^{\alpha},
$$

for all $s \in \mathcal{U}$. We name the number $f^{(\alpha)}(t)$ fractional derivative of order $\alpha$ at $t$. 
Besides the $\alpha$-differentiation theory in [3], an indefinite integral together with a number of firsthand properties are derived. However, some of those properties with their applicability and domain of validity have been discussed in [21].

Similar to Eqs. (2.6) and (2.7), if $f$ is continuous at $t$ and $t$ is right-scattered, then $f$ is differentiable of order $\alpha$ at $t$ with [3]

$$
f^{(\alpha)}(t)=\frac{f(\sigma(t))-f(t)}{\mu^{\alpha}(t)} .
$$

Let $\alpha \in(0,1] \cap\left\{\frac{1}{q} \mid \mathrm{q}\right.$ be odd $\}$. If $t$ is right-dense, then $f$ is fractional differentiable of order $\alpha$ at $t$ iff the limit

$$
f^{(\alpha)}(t):=\lim _{s \rightarrow t^{+}} \frac{f(t)-f(s)}{(t-s)^{\alpha}}
$$

exists and then it will be $f^{(\alpha)}(x)$ [3]. Similarly, if $\alpha \in(0,1] \cap\left\{\frac{1}{q} \mid q\right.$ is odd $\}$ and $t$ is left-dense. then $f$ is fractional differentiable of order $\alpha$ at $t$ iff the limit

$$
f^{(\alpha)}(t):=\lim _{s \longrightarrow t^{-}} \frac{f(t)-f(s)}{(t-s)^{\alpha}}
$$

exists and then it will be $f^{(\alpha)}(t)[3]$.

Remark 2.5 Reminding the notion of measure chain which leads us to the graininess function $\mu$, a fractional counterpart of it can be thought of as a pre-measure on a bent real line with the deflection parameter $\alpha$. In this way, a rough geometric interpretation of fractional derivative of a function at point $t \in \mathbb{T}$ of order $\alpha$ is a slope of a bent line tangent to the function at $t \in \mathbb{T}$.

Theorem 2.6 [5] Suppose $f, g: \mathbb{T} \rightarrow \mathbb{R}$ are pre-differentiable functions with $D$. Then

$$
\left|f^{\Delta}(t)\right| \leq g^{\Delta}(t), \quad \forall t \in D
$$

implies

$$
|f(s)-f(r)| \leq g(s)-g(r), \quad \forall r, s \in \mathbb{T}, r \leq s .
$$

Corollary 2.7 [5] Suppose $U=[r, s]$ or $[s, r]$, where $r, s \in \mathbb{T}$. If $f: \mathbb{T} \rightarrow \mathbb{R}$ is pre-differentiable functions with $D$, then the following inequality holds:

$$
|f(s)-f(r)| \leq\left\{\sup _{t \in U^{\kappa} \cap D}\left|f^{\Delta}(t)\right|\right\}|s-r| .
$$

A non-integer order counterpart of Theorem 2.6 is stated below:

Theorem 2.8 (Fractional Mean Value) Let $f, g: \mathbb{T} \rightarrow \mathbb{R}$ be $\alpha$-pre-differentiable functions with $D$. If

$$
\left|f^{(\alpha)}(t)\right| \leq g^{(\alpha)}(t), \quad \forall t \in D
$$

then

$$
|f(s)-f(r)| \leq g(s)-g(r), \quad \forall r, s \in \mathbb{T}, r \leq s .
$$

Proof The proof is based on the induction principle ([5], Theorem 1.7) and here it is given with a modification to the proof of Theorem 1.67 in [5].

Let $r, s \in \mathbb{T}$ with $r \leq s$. Since $\mathbb{T} \backslash D$ is countable, we denote it by $\left\{t_{n} \mid n \in \mathbb{N}\right\}$. Suppose the following statement

$S(t)$ : there exists a continuous and bounded function $M: \mathbb{T}^{\kappa} \rightarrow \mathbb{R}$ such that

$$
|f(t)-f(r)| \leq g(t)-g(r)+\epsilon\left(M(t)(t-r)^{\alpha}+\sum_{t_{n}<t} 2^{-n}\right) .
$$

The trivial case $S(r)$ is satisfied with any bounded and continuous $M$. Let $t$ be right-scattered, then $t \in D$ and assume that $S(t)$ holds. We have

$$
|f(\sigma(t))-f(r)|=\left|f(t)+\mu^{\alpha}(t) f^{(\alpha)}(t)-f(r)\right|
$$




$$
\begin{aligned}
& \leq \mu^{\alpha}(t)\left|f^{(\alpha)}(t)\right|+|f(t)-f(r)| \\
& \leq \mu^{\alpha}(t) g^{(\alpha)}(t)+g(t)-g(r)+\epsilon\left(M(t)(t-r)^{\alpha}+\sum_{t_{n}<t} 2^{-n}\right) \\
& \leq g(\sigma(t))-g(r)+\epsilon\left(M(t)(t-r)^{\alpha}+\sum_{t_{n}<\sigma(t)} 2^{-n}\right) .
\end{aligned}
$$

This shows that $S(\sigma(t))$ holds with $M(\sigma(t))=M(t)$.

Now, let $t$ be right-dense with $t \neq s$. First, suppose $t \in D$. Since $f$ and $g$ are pre-differentiable, then there exists a neighborhood $U$ of $t$ with

$$
\begin{aligned}
\left|f(t)-f(\tau)-(\tau-t)^{\alpha} f^{(\alpha)}(t)\right| & \leq \frac{\epsilon}{2}|\tau-t|^{\alpha} \quad \forall \tau \in U \\
\left|g(\tau)-g(t)-g^{(\alpha)}(t)(t-\tau)^{\alpha}\right| & \leq \frac{\epsilon}{2}|\tau-t|^{\alpha} \quad \forall \tau \in U
\end{aligned}
$$

This shows that, for $\tau \in U \cap(t, \infty)$, we have

$$
\begin{aligned}
|f(\tau)-f(r)| & \leq|f(\tau)-f(t)|+|f(t)-f(r)| \\
& \leq\left|f^{(\alpha)}(t)\right|(\tau-t)^{\alpha}+\frac{\epsilon}{2}(\tau-t)^{\alpha}+|f(t)-f(r)| \\
& \leq g^{(\alpha)}(t)(\tau-t)^{\alpha}+\frac{\epsilon}{2}(\tau-t)^{\alpha}+|f(t)-f(r)| \\
& \leq g(\tau)-g(t)+\epsilon(\tau-t)^{\alpha}+g(t)-g(r)+\epsilon\left(M(t)(t-r)^{\alpha}+\sum_{t_{n}<t} 2^{-n}\right) \\
& \leq g(\tau)-g(r)+\epsilon\left((\tau-r)^{\alpha}+M(t)(\tau-r)^{\alpha}+\sum_{t_{n}<\tau} 2^{-n}\right) .
\end{aligned}
$$

Therefore, the statement $S(\tau)$ for $\tau \in U \cap(t, \infty)$ holds with $M(\tau)=1+M(t)$.

Assume the other case, namely $t \in \mathbb{T} \backslash D$ or $t=t_{m}$ for some $m$. Since every pre-differentiable function is continuous, there exists a neighborhood $U$ of $t$ such that for every $\tau \in U$, we have

$$
|f(\tau)-f(t)| \leq \frac{\epsilon}{2} 2^{-m}, \quad g(\tau)-g(t) \geq \frac{\epsilon}{2} 2^{-m} .
$$

Hence,

$$
\begin{aligned}
|f(\tau)-f(r)| & \leq|f(\tau)-f(t)|+|f(t)-f(r)| \\
& \leq \frac{\epsilon}{2} 2^{-m}+g(t)-g(r)+\epsilon\left(M(t)(t-r)^{\alpha}+\sum_{t_{n}<t} 2^{-n}\right) \\
& \leq g(\tau)-g(r)+\epsilon\left(M(t)(\tau-r)^{\alpha}+\sum_{t_{n}<t} 2^{-n}\right) \\
& \leq g(\tau)-g(r)+\epsilon\left(M(t)(\tau-r)^{\alpha}+\sum_{t_{n}<\tau} 2^{-n}\right) .
\end{aligned}
$$

The last inequality holds since $t=t_{m}<\tau$. Therefore, $S(\tau)$ holds for all $\tau \in U \cap(t, \infty)$ with $M(\tau)=M(t)$. Now, let $t$ be a left-dense point and suppose $S(\tau)$ holds for $\tau<t$. Thus, we have

$$
\begin{aligned}
\lim _{\tau \rightarrow t^{-}}|f(\tau)-f(r)| & \leq \lim _{\tau \rightarrow t^{-}}\left\{g(\tau)-g(r)+\epsilon\left(M(\tau)(\tau-r)^{\alpha}+\sum_{t_{n}<\tau} 2^{-n}\right)\right\} \\
& \leq \lim _{\tau \rightarrow t^{-}}\left\{g(\tau)-g(r)+\epsilon\left(M(\tau)(\tau-r)^{\alpha}+\sum_{t_{n}<t} 2^{-n}\right)\right\},
\end{aligned}
$$

and $S(t)$ follows from continuity of $f, g$ and $M$ with $M(t)=\lim _{\tau \rightarrow t^{-}} M(\tau)$. We conclude that the above deductions prove the assertion. 


\section{Main results}

The content of this section evolves around the objectivity of the $\alpha$-derivative and it aims to show that the class of $\alpha$-differentiable functions with nonzero $\alpha$-derivative is rather small in the sense of smoothness. It is assumed that $\mathbb{T}$ is bounded and functions are continuous. Since the concentration is on the right-dense points, for the sake of objectivity, assume that the set of right-dense points is non-empty.

Proposition 3.1 Let $\mathbb{T}=[a, b]$ and assume that $f$ is analytic on a neighborhood of a point $x \in[a, b]$. Then $f^{(\alpha)} \equiv 0$.

Proof By the definition, we have

$$
\begin{aligned}
f^{(\alpha)}(x) & =\lim _{u \rightarrow 0} \frac{f\left(x+u^{1 / \alpha}\right)-f(x)}{u^{\alpha}} \\
& =\lim _{u \rightarrow 0} \sum_{n=1}^{\infty} f^{(n)}(x) u^{\frac{n-\alpha}{\alpha}}=0,
\end{aligned}
$$

where $(y-x)^{\alpha}=u$. Here, the uniform convergence of the series and uniform continuity of the family $\left(u^{\frac{n-\alpha}{\alpha}}\right)_{n}$ give the result.

Remark 3.2 The above result fails in the of lack of differentiability: Suppose $\mathbb{T}=\left\{t_{n} \mid n \in \mathbb{N}\right\} \cup\{t\}$ with $t_{n} \rightarrow t$ and let $f(t)=\left(t_{n}-t\right)^{\alpha}$, then $f^{(\alpha)}(t)=1$ while $t$ is dense.

Evidently, for a function satisfying $f(s)=O\left((s-t)^{\alpha}\right)$ when $s \rightarrow t$ implies $f^{(\alpha)}(t) \neq 0$ up to the dense points, but such asymptotic condition ruins the differentiability.

Proposition 3.3 Suppose that $\mathbb{T}^{\kappa^{n}} \neq \varnothing$ where $\mathbb{T}^{\kappa^{k+1}}=\left(\mathbb{T}^{\kappa^{k}}\right)^{\kappa}$. Assume that $f: \mathbb{T} \rightarrow \mathbb{R}$ is n-times continuously $\Delta$-differentiable. If $x \in \mathbb{T}^{\kappa^{n}}$ is a dense point, then $f^{(\alpha)}(x)=0$.

Proof According to [5], Theorem 1.111 (Taylor's Formula), since $g_{1}(y, x)^{\alpha}=(y-x)^{\alpha}$, then

$$
\begin{aligned}
f^{(\alpha)}(x)= & \lim _{y \rightarrow x} \frac{f(y)-f(x)}{(y-x)^{\alpha}} \\
= & \lim _{y \rightarrow x} \sum_{k=1}^{n-1} \frac{(-1)^{k} g_{k}(y, x)}{g_{1}(y, x)^{\alpha}} f^{\Delta^{k}}(x) \\
& +\lim _{y \rightarrow x} \int_{x}^{\rho^{n-1}(y)} \frac{(-1)^{n-1} g_{n-1}(\sigma(t), y)}{g_{1}(y, x)^{\alpha}} f^{\Delta^{n}}(t) \Delta t=0 . \quad n>1 .
\end{aligned}
$$

Indeed, from the definition of $g_{k}$, that is $g_{0}(y, x)=1$ and $g_{k+1}(y, x)=\int_{x}^{y} g_{k}(\sigma(t), x) \Delta t$, for $t \in\{t \in \mathbb{T} \mid x \leq$ $t<y$, by induction we obtain

$$
\frac{\int_{x}^{y} g_{k}(\sigma(t), x) \Delta t}{g_{1}(y, x)^{\alpha}} \leq \frac{(y-x)^{k+1}}{g_{1}(y, x)^{\alpha}}=(y-x)^{k-\alpha+1}, \quad k \in \mathbb{N}
$$

since $\sigma(t) \leq y$ and it completes the proof.

In favor of Theorem 2.8 , one can easily obtain:

Proposition 3.4 Let $f$ be a pre-differentiable function with $D$ and $t \in D$ be a (left or right)-dense point. If $f^{\Delta}$ is bounded on $D$, then $f^{(\alpha)}(t)=0$.

Corollary 3.5 Suppose $f \in C^{1}\left(\mathbb{T}^{\kappa}\right)$, then $f^{(\alpha)}$ vanishes at right-dense points.

Proof Assume $f^{(\alpha)}(t) \neq 0$ for some right-dense $t$. From Proposition 3.3, there exists a sequence $\left(t_{n}\right)_{n} \subset \mathbb{T}^{\kappa}$ with $f^{\Delta}\left(t_{n}\right) \rightarrow \infty$. Let $t_{0}=\lim t_{n}$, then $f$ is not $\Delta$-differentiable on $t_{0}$. It yields $t_{0} \notin \mathbb{T}^{\kappa}$ and therefore $t_{0}=\max \mathbb{T}$ and it contradicts the right-dense property. This proves the assertion. 
Definition 3.6 Let $E$ denote the set of right-dense points in $\mathbb{T}$. The set of all $\alpha$-pre-differentiable functions $f: \mathbb{T} \rightarrow \mathbb{R}$ for which $f^{(\alpha)}$ is bounded and non-zero on $E \cap D$, is denoted by $h_{+}^{\alpha}$. Also, $h_{0}^{\alpha}$ stands for the set of all $\alpha$-pre-differentiable functions with $f^{(\alpha)} \equiv 0$ on $E \cap D$ and $L i(\mathbb{T})$ denotes the space of Lipschitz functions. Clearly, $h_{+}^{\alpha}, h_{0}^{\alpha} \subset \mathcal{H}_{\alpha}(\mathbb{T})$, in which $\mathcal{H}_{\alpha}(\mathbb{T})$ is the the space of Hölder continuous functions on $\mathbb{T}$, which is a Banach space with the norm

$$
\|f\|_{\mathcal{H}_{\alpha}(\mathbb{T})}=\|f\|+\sup _{x, y \in \mathbb{T}}\left|\frac{f(x)-f(y)}{(x-y)^{\alpha}}\right|,
$$

in which $\|$.$\| is the sup norm.$

The following statement is immediate.

Proposition 3.7 $\operatorname{Li}(\mathbb{T}) \subset h_{0}^{\alpha}$.

Theorem 3.8 (Rademacher's Theorem, [24], Corollary 11.7) If $\Omega \subset \mathbb{R}^{n}$ is open and $f: \Omega \rightarrow \mathbb{R}^{m}$ is Lipschitz, then $f$ is differentiable almost everywhere.

As we already observed, there is no hope of looking inside the class of smooth functions for finding nontrivial $\alpha$-differentiable functions. If $\mathcal{N} \mathcal{D}([a, b])$ stands for the set of all nowhere-differentiable functions over $[a, b]$, then almost every function in $C[a, b]$ belongs to $\mathcal{N} \mathcal{D}[a, b]$ ([25], Theorem 4.8); hence, because of the prevalence of $\mathcal{N D}[a, b]$ in $C[a, b]$, it seems to be reasonable to regard the $\alpha$-derivative in connection with non-smooth analysis.

For the purpose of the following statement, let $Z\left(f^{(\alpha)}\right)$ be the set of zeros of $f^{(\alpha)}$.

Lemma 3.9 Let $\alpha, \beta \in \mathbb{R}^{+}$with $\alpha<\beta$. Then

1. $\mathcal{H}_{\beta}(\mathbb{T}) \subset \mathcal{H}_{\alpha}(\mathbb{T})$,

2. $\operatorname{Li}(\mathbb{T}) \cup h_{+}^{\alpha} \subset \mathcal{H}_{\alpha}(\mathbb{T})$,

3. $\operatorname{Li}(\mathbb{T}) \cap h_{+}^{\alpha}=\varnothing$,

4. Suppose $\mathbb{T}$ does not contain left-dense points. Assume that $f^{\Delta}$ is bounded on $\mathbb{T} \backslash E$ and $f \in h_{0}^{\alpha}$, then $f \in \operatorname{Li}(\mathbb{T})$.

Proof Let $x_{0} \in \mathbb{T}$ and assume that $f \in \mathcal{H}_{\beta}(\mathbb{T})$, then for the function $g_{x_{0}}$ defined by

$$
g_{x_{0}}(x)=\frac{f(x)-f\left(x_{0}\right)}{\left(x-x_{0}\right)^{\alpha}}
$$

we have $g_{x_{0}} \in \mathcal{H}_{\beta-\alpha}(\mathbb{T}) \subset C(\mathbb{T})$ and $\left\|g_{x_{0}}\right\|_{\mathcal{H}_{\beta-\alpha}(\mathbb{T})} \leq K\|f\|_{\mathcal{H}_{\beta}(\mathbb{T})}$, where $K$ does not depend on $f$ e.g., ([22] section 1.1). Since $g_{x_{0}}$ is continuous on $\mathbb{T}$, its limit exists at $x_{0}$ provided $x_{0} \in E$ and one can infer that $f$ is locally Hölder continuous. Also, by compactness of $\mathbb{T}$ and continuity of $g_{t}$ with respect to $t$, the family $\left\{g_{t}\right\}_{t \in \mathbb{T}}$ is uniform bounded and it proves $f \in \mathcal{H}_{\alpha}(\mathbb{T})$.

Parts 2 and 3 are clear.

4) Suppose $f \in h_{0}^{\alpha}$ and let $g(t)=\left(\sup _{t \in \mathbb{T} \backslash E}\left|f^{\Delta}(t)\right|\right) t$, then we have

$$
g^{(\alpha)}(t)=\left\{\begin{array}{lr}
0 & t \in E \\
\left(\sup _{t \in \mathbb{T} \backslash E}\left|f^{\Delta}(t)\right|\right) \mu^{1-\alpha}(t) & t \notin E
\end{array}\right.
$$

and therefore $\left|f^{(\alpha)}(t)\right| \leq g^{(\alpha)}(t)\left(t \in \mathbb{T}^{\kappa}\right)$ holds. The result is obvious from Theorem 2.8 since $g \in \operatorname{Li}(\mathbb{T})$.

Theorem 3.10 Suppose $f$ is almost nowhere differentiable, but $\alpha$-pre-differentiable with $D$.

1. There exists at least one point $t$ for which $f^{(\alpha)}(t) \neq 0$.

2. If $f^{(\alpha)}$ is continuous, then it does not vanish a.e.

Proof Part 1 is an immediate consequence of Theorem 2.8 .

2) Let $A=Z\left(f^{(\alpha)}\right)$ (zeros of $\left.f^{(\alpha)}\right)$, then $A \subset D$ is a timescale. By Theorem 2.8, $f$ is constant on $A$ and therefore $A$ has full measure. 


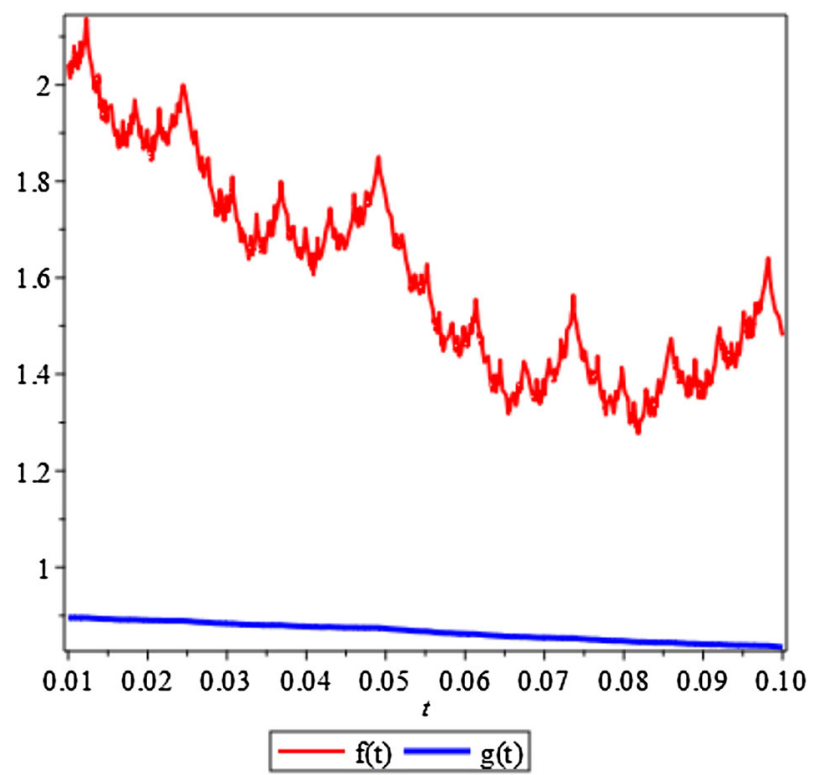

Fig. $1 f(t)$ and $g(t), \alpha=\frac{1}{2}, b=2$

\section{Non-differentiability vs $\alpha$-differentiability: examples}

Test Example 4.1 It is well known that the Weierstrass function defined by the following trigonometric series

$$
f(x)=\sum_{n=1}^{\infty} b^{-n \alpha} \cos b^{n} x, \quad 0<\alpha<1, b>1
$$

is non-differentiable and belongs to $\mathcal{H}_{\alpha}$. Fractal geometric study of the Weierstrass function (as a mono-fractal) and its generalization has an interesting literature, both in theory and in applications $[6,17,18,28]$.

Although in the present work, we have studied the local fractional derivative, non-local based fractional studies of $f$ are reported [23]. Indeed, there is a direct relationship between the order of Grunwald-Letnikov derivative of $f$ and its Hausdorff dimension [23].

Now, let $\left\{\epsilon_{n}\right\}_{n}$ be such that $\epsilon_{n} \rightarrow 0$, then the function

$$
g(x)=\sum_{n=1}^{\infty} \epsilon_{n} b^{-n \alpha} \cos b^{n} x
$$

has the following property:

$$
\sup |g(x)-g(y)|=o\left(|x-y|^{\alpha}\right),
$$

which leads to $g^{(\alpha)} \equiv 0$ (Chapter 2, Section 3, [29]). Then, we conclude that there exists a non-voided set upon which $g$ is differentiable (Fig. 1).

Test Example 4.2 ( $\boldsymbol{\alpha}$-Differentiability on $\boldsymbol{q}$-timescales)

Suppose $q \in] 0, \frac{\sqrt{5}-1}{2}\left[\right.$, and define $\mathbb{T}_{1}=\{0\} \cup q^{\mathbb{N}}=\left\{q^{n} \mid n \in \mathbb{N}\right\}, \mathbb{T}_{1}^{n}=\{0\} \cup q^{\mathbb{N}_{n+1}}, \mathbb{T}=\{1\} \cup \mathbb{T}_{1} \cup$ $\bigcup_{n \in \mathbb{N}}\left(q^{n}+\mathbb{T}_{1}^{n}\right)$. Let $l: \mathbb{R} \rightarrow \mathbb{R}$ be a periodic function defined by $l(t)=|t|(t \in[-1,1])$ and $l(t)=l\left(t+\frac{1}{q}\right)$. We define $f: \mathbb{T} \rightarrow \mathbb{R}$ by

$$
f(t)=\sum_{n \in \mathbb{N}_{0}} q^{n} l\left(q^{-n} t\right),
$$

which has a $q$-integral representation of the form (see $[10,19]$ for theory of integration and differentiation on q-timescales.)

$$
f(t)=\frac{q}{q-1} \int_{\mathbb{T}_{1} \cup\{1\}} l\left(\frac{t}{\tau}\right) d_{q} \tau, \quad t \in \mathbb{T}
$$




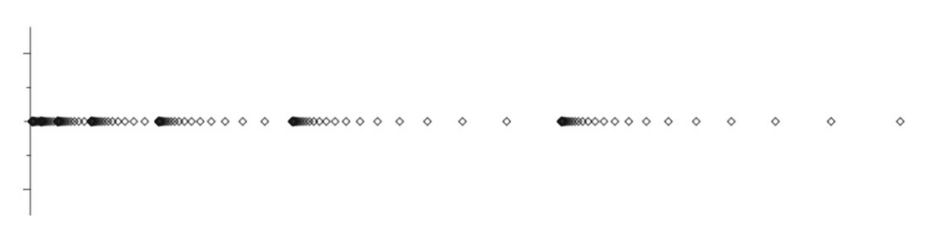

Fig. 2 Timescale $\tilde{\mathbb{T}}$

It is clear that $\mathbb{T}_{1} \subset \mathbb{T}$ consists of dense points of $\mathbb{T}$, and $f$ is $\Delta$-differentiable on $\mathbb{T} \backslash \mathbb{T}_{1}$. Let $t \in \mathbb{T}_{1}$, then $t=q^{k}$ for some $k \in \mathbb{N}$, and let $t_{j}=q^{j}+q^{k}$, in which $j>k$. Obviously, $\left.\left\{t_{j}\right\} \subset\right] q^{k}, q^{k-1}[$ since $q \in] 0, \frac{\sqrt{5}-1}{2}[$. Following that, it is easy to obtain

$$
f^{\Delta}(t)=\lim _{j \rightarrow \infty} \frac{f\left(q^{j}+q^{k}\right)-f\left(q^{k}\right)}{q^{j}}=\lim _{j \rightarrow \infty}\left(j+1+\frac{(j-k) q^{k}}{q^{j}}\right)=+\infty
$$

(since $h\left(q^{k} q^{-n}\right)=q^{k-n}$ when $n \leq k$ and otherwise $h\left(q^{k} q^{-n}\right)=0$ ). The same result holds for

$$
g(t)=\sum_{n \in \mathbb{N}_{0}} q^{n \alpha} l\left(q^{-n} t\right), \quad 0<\alpha<1, \quad t \in \mathbb{T},
$$

while we have

$$
\begin{aligned}
f^{\Delta}(t) & =\lim _{j \rightarrow \infty} \frac{\sum_{n=0}^{j} q^{n \alpha} \cdot q^{j-n}+\sum_{n=0}^{j} q^{n \alpha} \cdot q^{k-n}-\sum_{n=0}^{k} q^{n \alpha} \cdot q^{k-n}}{q^{j}} \\
& =\lim _{j \rightarrow \infty}\left(\sum_{n=0}^{j} q^{n(\alpha-1)}+\frac{q^{k}}{q^{j}} \sum_{n=k+1}^{j} q^{n(\alpha-1)}\right)=+\infty .
\end{aligned}
$$

Note that with the help of $\tilde{q}=q^{\alpha}$, Eq. (4.7) has a q-integral representation below

$$
g(t)=\frac{q^{\alpha}}{1-q^{\alpha}} \int_{q^{\alpha \mathbb{N}} \cup\{0,1\}} l\left(\frac{t}{\tau^{\frac{1}{\alpha}}}\right) d_{q} \tau, \quad t \in \tilde{\mathbb{T}},
$$

where $\tilde{\mathbb{T}}=\{0,1\} \cup q^{\alpha \mathbb{N}} \cup_{n \in \mathbb{N}} q^{\alpha n}+q^{\alpha \mathbb{N}_{n+1}}$ (Fig. 2). It can be verified that $g$ is not fractional differentiable of order $0<\beta<1$ on $\{0\} \cup q^{\alpha \mathbb{N}_{0}}$ :

$$
\begin{aligned}
f^{(\alpha)}(t) & =\lim _{j \rightarrow \infty} \frac{f\left(q^{j}++q^{k}\right)-f\left(+q^{k}\right)}{q^{j \beta}} \\
& =\lim _{j \rightarrow \infty} \frac{q^{\alpha-1}}{\left(q^{\alpha-1}-1\right) q^{j \beta}}\left(q^{j \alpha}-q^{j-\alpha+1}+q^{k-j+j \alpha}-q^{k \alpha}\right)=\infty .
\end{aligned}
$$

For the purpose of fractional differentiability, we need to modify the previous definitions: Let $l: \mathbb{R} \rightarrow \mathbb{R}$ be a differentiable function that vanishes outside $[0,1]$. We can define the corresponding function $h$ by

$$
h(t)=\sum_{n \in \mathbb{N}_{0}} q^{n \alpha} l\left(q^{-n} t^{\alpha}\right) . \quad 0<\alpha<1, \quad t \in \mathbb{T} .
$$

The summation in Eq. (4.11) is finite again, while $l$ vanishes outside of $[0,1]$, so for $t=q^{k}$, we have $l\left(q^{\alpha k-n}\right)=0$ for $\lfloor\alpha k\rfloor<n$, where $\lfloor$. $\rfloor$ is the floor function. The function $h$ is fractional differentiable of order $\alpha$, since for $t=q^{k}$ we have

$$
\begin{aligned}
h^{(\alpha)}(t) & =\lim _{j \rightarrow \infty} \frac{h\left(q^{j}+q^{k}\right)-h\left(q^{k}\right)}{q^{j \alpha}} \\
& =\lim _{j \rightarrow \infty} \frac{1}{q^{j \alpha}}\left(\sum_{n=0}^{\lfloor\alpha\rfloor} q^{n \alpha} l\left(q^{j \alpha-n}+q^{k \alpha-n}\right)-\sum_{n=0}^{k} q^{n \alpha} l\left(q^{k \alpha-n}\right)\right)
\end{aligned}
$$




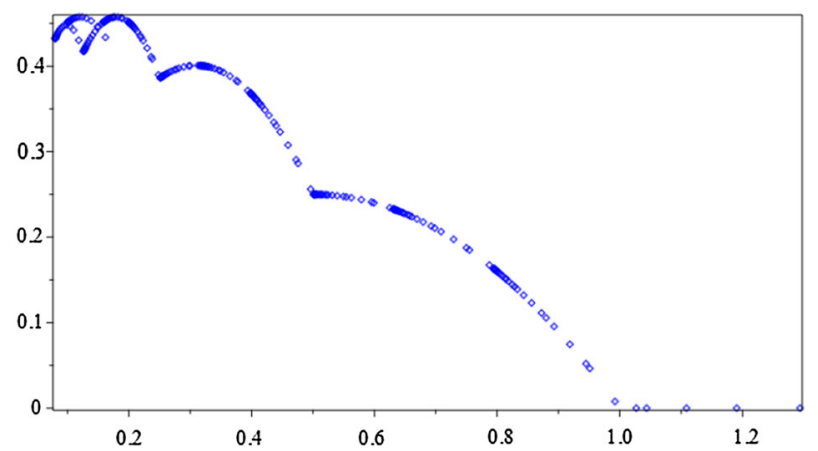

Fig. $3 h(t)$ as a function of $\tilde{\mathbb{T}}$ with $l(t)=t(1-t)$ for $t \in[0,1]$, otherwise $l(t)=0$, and $\alpha=\frac{1}{3}$

$$
\begin{aligned}
& =\sum_{n=0}^{\lfloor\alpha k\rfloor} q^{n \alpha} \lim _{j \rightarrow \infty} \frac{l\left(q^{j \alpha-n}+q^{k \alpha-n}\right)-l\left(q^{k \alpha-n}\right)}{q^{j \alpha}} \\
& =\sum_{n=0}^{\lfloor\alpha k\rfloor} q^{(\alpha+1) n} l^{\Delta}\left(q^{\alpha k-n}\right) .
\end{aligned}
$$

Note that one can obtain a similar result by considering $\tilde{\mathbb{T}}$ and defining

$$
h(t)=\sum_{n \in \mathbb{N}_{0}} q^{n \alpha} l\left(q^{-n} t\right) . \quad 0<\alpha<1, \quad t \in \tilde{\mathbb{T}} .
$$

Apparently, $h(t)$ in either of the above definitions is not $\Delta$-differentiable, but $\alpha$-differentiable on $\mathbb{T}$ and equivalently, $h$ as a function on $\tilde{\mathbb{T}}$ is $\Delta$-differentiable (see Fig. 3).

Remark 4.3 As it has been represented through the examples above, the discussed local non-integer order derivative and $\Delta$-derivative are equal up to a change of scale, i.e., changing $q^{\mathbb{N}_{0}}$ with $q^{\alpha \mathbb{N}_{0}}$ in Example 4.2. Interestingly, the transformation $t \longmapsto t^{\alpha}$ preserves the scattering property of two discussed timescales, that is, $\mu(t)=\frac{1-q}{q} t$ for $t \in \mathbb{T}$ and $\mu(t)=\frac{1-q^{\alpha}}{q^{\alpha}} t$ for $t \in \tilde{\mathbb{T}}$. The same invariance holds for $\mathbb{T}=\mathbb{Z}$ under the shift transformation $t \longmapsto t+\alpha$. In general, if a given timescale is invariant under a transformation (a change of scale as a function of $\alpha$ ), then we would be able to use $\Delta$ and $\alpha$ derivative interchangeably in the sense of $\mathbb{T}$ and $\tilde{\mathbb{T}}$ and their corresponding function $h(t)$.

\section{Conclusion}

In this work, an objectivity of the local $\alpha$-derivative on timescales has been discussed. Using the fractional mean value theorem, it is shown that, compared to the smooth functions, which have trivial $\alpha$-derivative, nowhere differentiable functions, which satisfy fractional differentiability, possess almost everywhere non-triviality of the fractional derivative of order $\alpha$. As it is shown in Example 4.1, the discussed local derivative has some potentials to study dynamics on self-similar fractals. The concepts of nowhere $\Delta$-differentiability up to dense points and $\alpha$-differentiability on timescales is also studied, and almost everywhere non-vanishing property of a fractional order derivative is stated. Making use of the series representation of q-integrals, a class of non-differentiable, but $\alpha$-differentiable on a q-timescale is introduced.

Open Access This article is distributed under the terms of the Creative Commons Attribution 4.0 International License (http:// creativecommons.org/licenses/by/4.0/), which permits unrestricted use, distribution, and reproduction in any medium, provided you give appropriate credit to the original author(s) and the source, provide a link to the Creative Commons license, and indicate if changes were made. 


\section{References}

1. Albrecht-Buehler, G.: Fractal genome sequences. Gene 498(1), 20-27 (2012)

2. Bastos, N.R.: Fractional calculus on time scales. Universidade de Aveiro, PhD thesis (2012)

3. Benkhettou, N.; da Cruz, A.M.B.; Torres, D.F.: A fractional calculus on arbitrary time scales: fractional differentiation and fractional integration. Signal Process. 107, 230-237 (2015)

4. Bohner, M.; Peterson, A.C. (eds.).: Advances in Dynamic Equations on Time Scales. Springer Science \& Business Media, New York (2002)

5. Bohner, M.; Peterson, A.: Dynamic Equations on Time Scales: An Introduction with Applications. Springer Science \& Business Media, New York (2001)

6. Carvalho, A.: Fractal geometry of Weierstrass-type functions. Fractals 17(01), 23-37 (2009)

7. Chen, W.: Time-space fabric underlying anomalous diffusion. Chaos Solitons Fract. 28(4), 923-929 (2006)

8. Chen, D.X.: Nonlinear oscillation of a class of second-order dynamic equations on time scales. Appl. Math. Sci. 6(60), 2957-2962 (2012)

9. Ciurdariu, L.: Integral inequalities on time scales via isotonic linear functionals. Appl. Math. Sci. 9(134), 6655-6668 (2015)

10. Ernst, T.: A Comprehensive Treatment of q-calculus. Springer Science \& Business Media (2012)

11. Falconer, K.: Fractal Geometry: Mathematical Foundations and Applications. Wiley, England (1990)

12. Golmankhaneh, A.K.; Baleanu, D.: Fractal calculus involving gauge function. Commun. Nonlinear Sci. Numer. Simul. 37, $125-130(2016)$

13. Guidolin, D.; Marinaccio, C.; Tortorella, C.; Ruggieri, S.; Rizzi, A.; Maiorano, E.; Specchia, G.; Ribatti, D.: A fractal analysis of the spatial distribution of tumoral mast cells in lymph nodes and bone marrow. Exp. Cell Res. 339(1), 96-102 (2015)

14. Havlin, S.; Buldyrev, S.V.; Goldberger, A.L.; Mantegna, R.N.; Ossadnik, S.M.; Peng, C.K.; Simons, M.; Stanley, H.E.: Fractals in biology and medicine. Chaos Solitons Fract. 6, 171-201 (1995)

15. Hilger, S.: Analysis on measure chains-a unified approach to continuous and discrete calculus. Results Math. 18(1-2), 18-56 (1990)

16. Hilger, S.: Differential and difference calculus-unified!. Nonlinear Anal. Theory Methods Appl. 30(5), 2683-2694 (1997)

17. Humphrey, J.A.C.; Schuler, C.A.; Rubinsky, B.: On the use of the Weierstrass-Mandelbrot function to describe the fractal component of turbulent velocity. Fluid Dyn. Res. 9(1-3), 81-95 (1992)

18. Jiang, S.; Zheng, Y.: An analytical model of thermal contact resistance based on the Weierstrass-Mandelbrot fractal function. In: Proceedings of the Institution of Mechanical Engineers, Part C: Journal of Mechanical Engineering Science 224(4), 959-967 (2010)

19. Kac, V.; Cheung, P.: Quantum Calculus. Springer, Berlin (2002)

20. Liang, Y.; Allen, Q.Y.; Chen, W.; Gatto, R.G.; Colon-Perez, L.; Mareci, T.H.; Magin, R.L.: A fractal derivative model for the characterization of anomalous diffusion in magnetic resonance imaging. Commun. Nonlinear Sci. Numer. Simul. 39, 529-537 (2016)

21. Neamaty, A.; Nategh, M.; Agheli, B.: Time-space fractional Burger's equation on time scales. J. Comput. Nonlinear Dyn. 12(3), $031022(2017)$

22. Samko, S.G.; Kilbas, A.A.; Marichev, O.I.: Fractional Integrals and Derivatives. Theory and Applications. Gordon and Breach, Yverdon (1993)

23. Stiassnie, M.: A look at fractal functions through their fractional derivatives. Fractals 5(04), 561-564 (1997)

24. Taylor, M.E.: Measure Theory and Integration. American Mathematical Society, Rhode Island (2006)

25. Thim, J.: Continuous nowhere differentiable functions. Lule $\dot{a}$ University. Master Thesis (2003)

26. Williams, P.A.: Unifying fractional calculus with time scales. University of Melbourne, PhD thesis (2012)

27. Yang, X.J.; Baleanu, D.; Srivastava, H.M.: Local Fractional Integral Transforms and Their Applications. Elsevier, London (2015)

28. Zhang, L.; Yu, C.; Sun, J.Q.: Generalized weierstrass-mandelbrot function model for actual stocks markets indexes with nonlinear characteristics. Fractals 23(02), 1550006 (2015)

29. Zygmund, A.: Trigonometric Series. Volume I \& II combined, 3rd edn. Cambridge University Press, Cambridge (2002)

\section{Publisher's Note}

Springer Nature remains neutral with regard to jurisdictional claims in published maps and institutional affiliations. 